
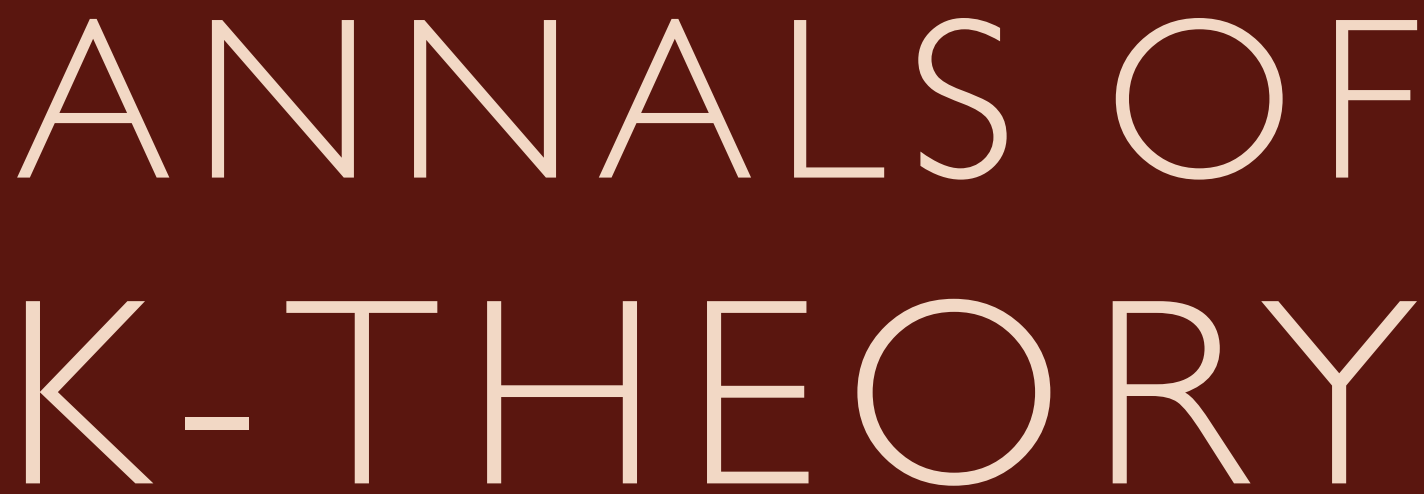

vol. 2 no. 32017

Colocalising subcategories of modules over finite group schemes

Dave Benson, Srikanth B. Iyengar, Henning Krause and Julia Pevtsova

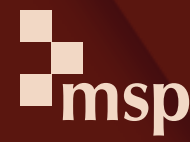
A JOURNAL
OF THE K-THEO 


\title{
Colocalising subcategories of modules over finite group schemes
}

\author{
Dave Benson, Srikanth B. Iyengar, Henning Krause and Julia Pevtsova
}

Dedicated to Amnon Neeman on the occasion of his 60th birthday

\begin{abstract}
The Hom closed colocalising subcategories of the stable module category of a finite group scheme are classified. This complements the classification of the tensor closed localising subcategories in our previous work. Both classifications involve $\pi$-points in the sense of Friedlander and Pevtsova. We identify for each $\pi$-point an endofinite module which both generates the corresponding minimal localising subcategory and cogenerates the corresponding minimal colocalising subcategory.
\end{abstract}

\section{Introduction}

Let $G$ be a finite group scheme over a field $k$ of positive characteristic. There is a notion of $\pi$-cosupport [Benson et al. 2017] for any $G$-module $M$, based on the notion of $\pi$-points of $G$ introduced by Friedlander and Pevtsova [2005]. The $\pi$-cosupport of $M$, denoted by $\pi$-cosupp $(M)$, is a subset of Proj $H^{*}(G, k)$. The main result in this work is a classification of the colocalising subcategories of StMod $G$, the stable module category of possibly infinite-dimensional $G$-modules, in terms of $\pi$-cosupport.

Theorem 1.1. Let $G$ be a finite group scheme over a field $k$. Then the assignment

$$
\mathrm{C} \mapsto \bigcup_{M \in \mathrm{C}} \pi-\operatorname{cosupp}_{G}(M)
$$

induces a bijection between the colocalising subcategories of StMod $G$ that are closed under tensor product with simple G-modules and the subsets of Proj $H^{*}(G, k)$.

This is proved after Corollary 4.9. Recall that a colocalising subcategory $\mathrm{C}$ is a full triangulated subcategory that is closed under set-indexed products. Such a $\mathrm{C}$ is closed under tensor product with simple $G$-modules if and only if it is Hom closed: if $M$ is in C, so is $\operatorname{Hom}_{k}(L, M)$ for any $G$-module $L$. Theorem 1.1 complements

Iyengar was partly supported by NSF grant DMS-1503044 and Pevtsova was partly supported by NSF grants DMS-0953011 and DMS-1501146.

MSC2010: primary 16G10; secondary 18E30, 20C20, 20G10, $20 \mathrm{~J} 06$.

Keywords: cosupport, stable module category, finite group scheme, colocalising subcategory. 
the classification of the tensor closed localising subcategories of StMod $G$ from [Benson et al. 2016]. Combining them gives a remarkable bijection:

Corollary 1.2. The map sending a localising subcategory $\mathrm{C}$ of $\mathrm{StMod} G$ to $\mathrm{C}^{\perp}$ induces a bijection

$$
\left\{\begin{array}{c}
\text { tensor closed localising } \\
\text { subcategories of StMod } G
\end{array}\right\} \stackrel{\sim}{\rightarrow}\left\{\begin{array}{c}
\text { Hom closed colocalising } \\
\text { subcategories of StMod } G
\end{array}\right\} .
$$

The inverse map sends a colocalising subcategory $\mathrm{C}$ to ${ }^{\perp} \mathrm{C}$.

Predecessors of these results are the analogues for the derived category of a commutative noetherian ring by Neeman [2011], and the stable module category of a finite group [Benson et al. 2012]. Any finite group gives rise to a finite group scheme, and we obtain an entirely new proof in that case.

Products of modules tend to be more complicated than coproducts. This is reflected by the fact that the classification of colocalising subcategories formally implies the classification of localising subcategories in terms of $\pi$-supports of $G$ modules; see [Benson et al. 2012, Theorem 9.7]. So Theorem 1.1 implies the classification result in our work presented in [Benson et al. 2016]. However, the arguments in the present work rely heavily on the tools developed in [loc. cit.], which, in turn, depend on the fundamental results and geometric techniques for the representation theory and cohomology of finite group schemes from [Suslin 2006; Suslin et al. 1997].

An essential ingredient in the proof of Theorem 1.1 is a family of $G$-modules, one arising from each $\pi$-point of $G$. We call them point modules and write $\Delta_{G}(\alpha)$, where $\alpha: K[t] /\left(t^{p}\right) \rightarrow K G$ is the corresponding $\pi$-point. They appear already in [Benson et al. 2016, Section 9] and play the role of residue fields in commutative algebra. Indeed, while they are not usually finite-dimensional, they are always endofinite in the sense of Crawley-Boevey [1991], as we prove in Proposition 3.8. It follows from results in [Benson et al. 2016] that the $\pi$-support of $\Delta_{G}(\alpha)$ is equal to the prime ideal $\mathfrak{p}$ corresponding to $\alpha$, and that the localising subcategory generated by $\Delta_{G}(\alpha)$ is $\Gamma_{\mathfrak{p}}$ StMod $G$, the full subcategory of $\mathfrak{p}$-local and $\mathfrak{p}$-torsion objects.

In Theorem 4.4, we prove that $\Delta_{G}(\alpha)$ also cogenerates $\Lambda^{\mathfrak{p}}$ StMod $G$, the full subcategory of $\mathfrak{p}$-local and $\mathfrak{p}$-complete $G$-modules, in the sense of [Benson et al. 2012]. This result is an important step in the proof of Theorem 1.1, because the subcategories $\Lambda^{\mathfrak{p}}$ StMod $G$, as $\mathfrak{p}$ varies over Proj $H^{*}(G, k)$, cogenerate StMod $G$. From this it follows that every Hom closed colocalising subcategories of StMod $G$ is cogenerated by point modules, which again highlights the special role played by them.

There is a parallel between point modules and standard objects in highest weight categories studied by Cline, Parshall and Scott [Cline et al. 1988]. This is explained towards the end of this article. The notation $\Delta_{G}(\alpha)$ reflects this connection. 


\section{Recollections}

In this section we recall basic notions and results we will need about modules over finite group schemes. Our standard references are [Jantzen 2003; Waterhouse 1979]. For the later parts, and for the notation, we follow [Benson et al. 2016].

Let $G$ be a finite group scheme over a field $k$. Thus $G$ is an affine group scheme such that its coordinate algebra $k[G]$ is finite-dimensional as a $k$-vector space. The $k$-linear dual of $k[G]$ is a cocommutative Hopf algebra, called the group algebra of $G$, and denoted by $k G$. We identify $G$-modules with modules over the group algebra $k G$. The category of all (left) $G$-modules is denoted by Mod $G$.

The stable module category StMod $G$ is obtained from Mod $G$ by identifying two morphisms between $G$-modules when they factor through a projective $G$-module. The tensor product of $G$-modules passes to StMod $G$ and we obtain a compactly generated tensor triangulated category with suspension $\Omega^{-1}$, the inverse of the syzygy functor. We use the notation $\underline{\operatorname{Hom}}_{G}(M, N)$ for the Hom-sets in StMod $G$. For details, readers might consult [Carlson 1996, §5; Happel 1988, Chapter 1].

In the context of finite groups there is a duality theorem due to Tate [Cartan and Eilenberg 1956, Chapter XII, Theorem 6.4] that is helpful in computing morphisms in the stable category. In the proof of Lemma 4.1 we need an extension of this to finite group schemes, which is recalled below.

Duality. Given a $k$-vector space $V$, we set $V^{\vee}:=\operatorname{Hom}_{k}(V, k)$ to be the dual vector space. If $V$ is a $G$-module, then $V^{\vee}$ can also be endowed with a structure of a $G$ module using the Hopf algebra structure of $k G$.

Let $G^{\text {op }}$ denote the opposite group scheme that is given by the group algebra $(k G)^{\mathrm{op}}$. Given a $G^{\mathrm{op}}$-module $M$, we write $D M:=\operatorname{Hom}_{k}(M, k)$ for the dual vector space considered as a $G$-module. Let

$$
\tau=D \circ \operatorname{Tr}: \operatorname{stmod} G \stackrel{\sim}{\longrightarrow} \operatorname{stmod} G
$$

be the composition of the duality functor $D$ and the transpose

$$
\operatorname{Tr}: \operatorname{stmod} G \rightarrow \operatorname{stmod} G^{\text {op }}
$$

see [Skowroński and Yamagata 2011, Section III.4] for the definition. For any $G$ module $M$ and finite-dimensional $G$-module $N$, there is a natural isomorphism of vector spaces

$$
\underline{\operatorname{Hom}}_{G}(N, M)^{\vee} \cong \underline{\operatorname{Hom}}_{G}\left(M, \Omega^{-1} \tau N\right) .
$$

This isomorphism can be deduced from a formula of Auslander and Reiten [Auslander 1978, Proposition I.3.4] — see also [Krause 2003, Corollary, p. 269] — which yields the first isomorphism below:

$$
\underline{\operatorname{Hom}}_{G}(N, M)^{\vee} \cong \operatorname{Ext}_{G}^{1}(M, \tau N) \cong \underline{\operatorname{Hom}}_{G}\left(M, \Omega^{-1} \tau N\right) .
$$


When $k G$ is symmetric (in particular, whenever $G$ is a finite group), we have $\tau N \cong \Omega^{2} N$. This follows from [Skowroński and Yamagata 2011, Section IV.8] and reduces (2.1) to Tate duality.

Extending the base field. Let $G$ be a finite group scheme over a field $k$. If $K$ is a field extension of $k$, we write $K[G]$ for $K \otimes_{k} k[G]$, which is a commutative Hopf algebra over $K$. This defines a finite group scheme over $K$, denoted by $G_{K}$. We have a natural isomorphism $K G_{K} \cong K \otimes_{k} k G$ and we simplify notation by writing $K G$. The restriction functor

$$
\operatorname{res}_{k}^{K}: \operatorname{Mod} G_{K} \rightarrow \operatorname{Mod} G
$$

admits a left adjoint that sends a $G$-module $M$ to

$$
M_{K}:=K \otimes_{k} M,
$$

and a right adjoint sending $M$ to

$$
M^{K}:=\operatorname{Hom}_{k}(K, M) .
$$

The next result tracks how these functors interact with taking tensors and modules of homomorphisms. We give proofs for lack of an adequate reference.

Lemma 2.2. Let $K$ be a field extension of $k$. For $a G_{K}$-module $M$ and $a G$ module $N$, there are natural isomorphisms of $G$-modules

$$
\begin{aligned}
\operatorname{res}_{k}^{K}\left(M \otimes_{K} N_{K}\right) & \cong\left(\operatorname{res}_{k}^{K} M\right) \otimes_{k} N, \\
\operatorname{res}_{k}^{K} \operatorname{Hom}_{K}\left(M, N^{K}\right) & \cong \operatorname{Hom}_{k}\left(\operatorname{res}_{k}^{K} M, N\right) .
\end{aligned}
$$

Proof. The first isomorphism is clear since the $k$-linear isomorphism

$$
M \otimes_{K}\left(K \otimes_{k} N\right) \cong\left(M \otimes_{K} K\right) \otimes_{k} N \cong M \otimes_{k} N
$$

is compatible with the diagonal $G$-actions.

The second isomorphism follows from the first one, because the functor

$$
\operatorname{res}_{k}^{K} \operatorname{Hom}_{K}\left(M,(-)^{K}\right) \text { is right adjoint to } \operatorname{res}_{k}^{K}\left(M \otimes_{K}(-)_{K}\right),
$$

while the functor

$$
\operatorname{Hom}_{k}\left(\operatorname{res}_{k}^{K} M,-\right) \text { is right adjoint to }\left(\operatorname{res}_{k}^{K} M\right) \otimes_{k}-\text {. }
$$

Subgroup schemes. For each subgroup scheme $H$ of $G$ restriction is a functor

$$
\operatorname{res}_{H}^{G}: \operatorname{Mod} G \rightarrow \operatorname{Mod} H .
$$

This has a right adjoint, called induction,

$$
\operatorname{ind}_{H}^{G}: \operatorname{Mod} H \rightarrow \operatorname{Mod} G,
$$


as described in [Jantzen 2003, Section I.3.3], and a left adjoint, called coinduction,

$$
\operatorname{coind}_{H}^{G}: \operatorname{Mod} H \rightarrow \operatorname{Mod} G
$$

as described in [Jantzen 2003, Section I.8.14].

Lemma 2.3. Let $H$ be a subgroup scheme of $G$. For any $H$-module $M$ and $G$ module $N$ there are natural isomorphisms

$$
\begin{aligned}
\operatorname{coind}_{H}^{G}\left(M \otimes_{k} \operatorname{res}_{H}^{G} N\right) \cong\left(\operatorname{coind}_{H}^{G} M\right) \otimes_{k} N, \\
\operatorname{ind}_{H}^{G} \operatorname{Hom}_{k}\left(M, \operatorname{res}_{H}^{G} N\right) \cong \operatorname{Hom}_{k}\left(\operatorname{coind}_{H}^{G} M, N\right) .
\end{aligned}
$$

In particular, for $M=k$ these give isomorphisms

$$
\begin{aligned}
\operatorname{coind}_{H}^{G} \operatorname{res}_{H}^{G} N \cong\left(\operatorname{coind}_{H}^{G} k\right) \otimes_{k} N, \\
\operatorname{ind}_{H}^{G} \operatorname{res}_{H}^{G} N \cong \operatorname{Hom}_{k}\left(\operatorname{coind}_{H}^{G} k, N\right) .
\end{aligned}
$$

Proof. Recalling that coind ${ }_{H}^{G}=k G \otimes_{k H}-$, the first isomorphism follows from associativity of tensor products:

$$
\begin{aligned}
\operatorname{coind}_{H}^{G}\left(M \otimes_{k} \operatorname{res}_{H}^{G} N\right) & \cong k G \otimes_{k H}\left(M \otimes_{k} \operatorname{res}_{H}^{G} N\right) \\
& \cong\left(k G \otimes_{k H} M\right) \otimes_{k} N \\
& \cong\left(\operatorname{coind}_{H}^{G} M\right) \otimes_{k} N .
\end{aligned}
$$

The second isomorphism follows from the first one, because the functor $\operatorname{ind}_{H}^{G} \operatorname{Hom}_{k}(M,-) \operatorname{res}_{H}^{G}$ is right adjoint to $\operatorname{coind}_{H}^{G}\left(M \otimes_{k}-\right) \operatorname{res}_{H}^{G}$,

while the functor

$$
\operatorname{Hom}_{k}\left(\operatorname{coind}_{H}^{G} M,-\right) \text { is right adjoint to }\left(\operatorname{coind}_{H}^{G} M\right) \otimes_{k}-\text {. }
$$

Cohomology and $\pi$-points. Let $k$ be a field of positive characteristic $p$ and $G$ a finite group scheme over $k$. We write $H^{*}(G, k)$ for the cohomology algebra of $G$ and $\operatorname{Proj} H^{*}(G, k)$ for the set of its homogeneous prime ideals not containing $H^{\geqslant 1}(G, k)$, the elements of positive degree.

A $\pi$-point of $G$, defined over a field extension $K$ of $k$, is a morphism of $K$ algebras

$$
\alpha: K[t] /\left(t^{p}\right) \rightarrow K G
$$

that factors through the group algebra of a unipotent abelian subgroup scheme of $G_{K}$, and such that $K G$ is flat when viewed as a left (equivalently, as a right) module over $K[t] /\left(t^{p}\right)$ via $\alpha$. Given such an $\alpha$, restriction yields a functor

$$
\alpha^{*}: \operatorname{Mod} K G \rightarrow \operatorname{Mod} K[t] /\left(t^{p}\right) .
$$


We write $H^{*}(\alpha)$ for the composition of homomorphisms of $k$-algebras

$$
H^{*}(G, k)=\operatorname{Ext}_{G}^{*}(k, k) \stackrel{K \otimes_{k}-}{\longrightarrow} \operatorname{Ext}_{G_{K}}^{*}(K, K) \stackrel{\alpha^{*}}{\longrightarrow} \operatorname{Ext}_{K[t] /\left(t^{p}\right)}^{*}(K, K) .
$$

The radical of the ideal $\operatorname{Ker} H^{*}(\alpha)$ is a prime ideal in $H^{*}(G, k)$, and the assignment $\alpha \mapsto \sqrt{\operatorname{Ker} H^{*}(\alpha)}$ yields a bijection between the equivalence classes of $\pi$-points and Proj $H^{*}(G, k)$; see [Friedlander and Pevtsova 2007, Theorem 3.6]. Recall that $\pi$-points $\alpha: K[t] /\left(t^{p}\right) \rightarrow K G$ and $\beta: L[t] /\left(t^{p}\right) \rightarrow L G$ are equivalent if for every $G$ module $M$ the module $\alpha^{*}\left(M_{K}\right)$ is projective if and only if $\beta^{*}\left(M_{L}\right)$ is projective. In the sequel, we identify a prime in $\operatorname{Proj} H^{*}(G, k)$ and the corresponding equivalence class of $\pi$-points.

Given a point in Proj $H^{*}(G, k)$, there is some flexibility in choosing a $\pi$-point representing it. This will be important in the sequel.

Remark 2.4. We call a group scheme $\mathscr{E}$ quasielementary if there is an isomorphism $\mathscr{E} \cong \mathbb{G}_{a(r)} \times E$, where $\mathbb{G}_{a(r)}$ is the $r$-th Frobenius kernel of the additive group $\mathbb{G}_{a}$ and $E$ is an elementary abelian $p$-group.

By Proposition 4.2 of [Friedlander and Pevtsova 2005], given a $\pi$-point $\alpha$ : $K[t] /\left(t^{p}\right) \rightarrow K G$, there exists an equivalent $\pi$-point $\beta: K[t] /\left(t^{p}\right) \rightarrow K G$ that factors through a quasielementary subgroup scheme of $G_{K}$.

A point $\mathfrak{p}$ in Proj $H^{*}(G, k)$ is closed if there is no point in Proj $H^{*}(G, k)$ properly containing it as a prime ideal. Then there exists a $\pi$-point $\alpha: K[t] /\left(t^{p}\right) \rightarrow K G$ such that $K$ is finite-dimensional over $k$; see Theorem 4.2 of [Friedlander and Pevtsova 2007]. In view of the preceding paragraph, one may choose an $\alpha$ that factors through a quasielementary subgroup scheme of $G_{K}$.

Local cohomology and completions. We recall from [Benson et al. 2008; 2012] the definition of local cohomology and completion for $G$-modules.

The algebra $H^{*}(G, k)$ acts on StMod $G$. This means that for $G$-modules $M$ and $N$ there is a natural action of $H^{*}(G, k)$ on

$$
\underline{\operatorname{Hom}}_{G}^{*}(M, N)=\bigoplus_{i \in \mathbb{Z}} \underline{\operatorname{Hom}}_{G}\left(\Omega^{i} M, N\right)
$$

via the homomorphism of $k$-algebras

$$
-\otimes_{k} M: H^{*}(G, k)=\operatorname{Ext}_{G}^{*}(k, k) \rightarrow \underline{\operatorname{Hom}}_{G}^{*}(M, M) .
$$

Fix $\mathfrak{p} \in \operatorname{Proj} H^{*}(G, k)$. There is a localisation functor StMod $G \rightarrow \operatorname{StMod} G$ sending $M$ to $M_{\mathfrak{p}}$ such that the natural morphism $M \rightarrow M_{\mathfrak{p}}$ induces an isomorphism

$$
\underline{\operatorname{Hom}}_{G}^{*}(-, M)_{\mathfrak{p}} \stackrel{\sim}{\longrightarrow} \underline{\operatorname{Hom}}_{G}^{*}\left(-, M_{\mathfrak{p}}\right)
$$

when restricted to finite-dimensional $G$-modules. A $G$-module $M$ is called $\mathfrak{p}$-local if $M \stackrel{\sim}{\rightarrow} M_{\mathfrak{p}}$, and we write (StMod $\left.G\right)_{\mathfrak{p}}$ for the full subcategory of $\mathfrak{p}$-local $G$ modules. The module $M$ is $\mathfrak{p}$-torsion if $M_{\mathfrak{q}}=0$ for all $\mathfrak{q} \in \operatorname{Spec} H^{*}(G, k)$ that do not 
contain $\mathfrak{p}$. There is a colocalisation functor $\Gamma_{\mathscr{V}(\mathfrak{p})}:$ StMod $G \rightarrow$ StMod $G$ such that the natural morphism $\Gamma_{\mathscr{V}(\mathfrak{p})}(M) \rightarrow M$ is an isomorphism if and only $M$ is $\mathfrak{p}$-torsion. The functor $\Gamma_{\mathscr{V}(\mathfrak{p})}$ admits a right adjoint, denoted by $\Lambda^{\mathscr{V}(\mathfrak{p})}$ and called $\mathfrak{p}$-completion. We say that $M$ is $\mathfrak{p}$-complete if the natural map $M \rightarrow \Lambda^{\mathscr{V}(\mathfrak{p})} M$ is an isomorphism.

The functor $\Gamma_{\mathfrak{p}}:$ StMod $G \rightarrow \operatorname{StMod} G$ sending $M$ to $\Gamma_{\mathscr{V}(\mathfrak{p})}\left(M_{\mathfrak{p}}\right)$ gives local cohomology at $\mathfrak{p}$. It has a right adjoint $\Lambda^{\mathfrak{p}}: \operatorname{StMod} G \rightarrow \operatorname{StMod} G$ that plays the role of completion at $\mathfrak{p}$ for modules over commutative rings.

Koszul objects and reduction to closed points. Given a cohomology class $\zeta$ in $H^{*}(G, k)$, let $k / / \zeta$ be a mapping cone of the morphism $k \rightarrow \Omega^{-d} k$ in StMod $G$ defined by $\zeta$. Note that $k / / \zeta \cong \Omega^{-d-1} L_{\zeta}$, where $L_{\zeta}$ is the Carlson module [1983] defined by $\zeta$. For a homogeneous ideal $\mathfrak{a}$ in $H^{*}(G, k)$, we pick a system of homogeneous generators $\zeta_{1}, \ldots, \zeta_{n}$, and define a Koszul object $k / / \mathfrak{a}$ to be

$$
k / / \mathfrak{a}:=k / / \zeta_{1} \otimes_{k} \cdots \otimes_{k} k / / \zeta_{n} .
$$

Observe that the map $k \rightarrow \Omega^{-d} k$ defined by $\zeta$ becomes an isomorphism when localised at any prime ideal $\mathfrak{p}$ of $H^{*}(G, k)$ not containing $\zeta$. Given this, the next result is [Benson et al. 2016, Theorem 8.8].

Theorem 2.5. Let $\mathfrak{p}$ be a point in Proj $H^{*}(G, k)$. There exists a field extension $L / k$ and an ideal $\mathfrak{q}$ of $H^{*}\left(G_{L}, L\right)$ with radical $\sqrt{\mathfrak{q}}$ a closed point in Proj $H^{*}\left(G_{L}, L\right)$ lying over $\mathfrak{p}$ such that there is an isomorphism

$$
\operatorname{res}_{k}^{L}(L / / \mathfrak{q}) \cong(k / / \mathfrak{p})_{\mathfrak{p}} .
$$

The construction of $L / / \mathfrak{q}$ involves a choice of generators for $\mathfrak{q}$, so the theorem effectively states that there exist an ideal $\mathfrak{q}$ and a choice of generators that produces the Koszul object with required properties. For details, see [Benson et al. 2016, Section 8].

Brown representability. Let $C$ be a finite-dimensional $G$-module and $I$ an injective $H^{*}(G, k)$-module. Recall that $H^{*}(G, k)$ acts on $\underline{\operatorname{Hom}}_{G}^{*}(C, M)$ for any $M$ in StMod $G$, and consider the contravariant functor

$$
\operatorname{Hom}_{H^{*}(G, k)}\left(\underline{\operatorname{Hom}}_{G}^{*}(C,-), I\right): \operatorname{StMod} G \rightarrow \mathrm{Ab}
$$

This functor takes triangles to exact sequences and coproducts to products. Hence, by the contravariant version of Brown representability (see [Brown 1965] or [Neeman 1996]), there exists a $G$-module $T_{C}(I)$ such that

$$
\operatorname{Hom}_{H^{*}(G, k)}\left(\underline{\operatorname{Hom}}_{G}^{*}(C,-), I\right) \cong \underline{\operatorname{Hom}}_{G}\left(-, T_{C}(I)\right) .
$$

We refer to [Benson et al. 2012; Benson and Krause 2002] for details about these modules. 
Support and cosupport. The following definitions of $\pi$-support and $\pi$-cosupport of a $G$-module $M$ are from [Friedlander and Pevtsova 2007] and [Benson et al. 2017], respectively. We set

$$
\begin{aligned}
\pi-\operatorname{supp}_{G}(M) & :=\left\{\mathfrak{p} \in \operatorname{Proj} H^{*}(G, k) \mid \alpha_{\mathfrak{p}}^{*}\left(M_{K}\right) \text { is not projective }\right\}, \\
\pi-\operatorname{cosupp}_{G}(M) & :=\left\{\mathfrak{p} \in \operatorname{Proj} H^{*}(G, k) \mid \alpha_{\mathfrak{p}}^{*}\left(M^{K}\right) \text { is not projective }\right\} .
\end{aligned}
$$

Here $\alpha_{\mathfrak{p}}: K[t] /\left(t^{p}\right) \rightarrow K G$ denotes a $\pi$-point corresponding to $\mathfrak{p}$. Both $\pi$-supp and $\pi$-cosupp are well-defined on the equivalence classes of $\pi$-points [Benson et al. 2017, Theorem 3.1].

The local cohomology functors $\Gamma_{\mathfrak{p}}$ and their right adjoints $\Lambda^{\mathfrak{p}}$ yield alternative notions of support and cosupport for a $G$-module $M$; see [Benson et al. 2008; Benson et al. 2012]. We set

$$
\begin{aligned}
\operatorname{supp}_{G}(M) & :=\left\{\mathfrak{p} \in \operatorname{Proj} H^{*}(G, k) \mid \Gamma_{\mathfrak{p}} M \neq 0\right\}, \\
\operatorname{cosupp}_{G}(M) & :=\left\{\mathfrak{p} \in \operatorname{Proj} H^{*}(G, k) \mid \Lambda^{\mathfrak{p}} M \neq 0\right\} .
\end{aligned}
$$

It is an important fact that these notions agree with the ones defined via $\pi$-points. This will be used freely throughout this work.

Theorem 2.7 [Benson et al. 2016, Theorems 6.1 and 9.3]. For every $G$-module $M$ there are equalities

$$
\pi-\operatorname{supp}_{G}(M)=\operatorname{supp}_{G}(M) \text { and } \pi-\operatorname{cosupp}_{G}(M)=\operatorname{cosupp}_{G}(M) .
$$

For ease of reference we recall basic facts concerning support and cosupport.

Remark 2.8. Let $M$ and $N$ be $G$-modules.

(1) $M$ is projective if and only if $\operatorname{supp}_{G}(M)=\varnothing$ if and only if $\operatorname{cosupp}_{G}(M)=\varnothing$.

(2) $\operatorname{supp}_{G}(M)$ and $\operatorname{cosupp}_{G}(M)$ have the same maximal elements with respect to inclusion.

(3) $\operatorname{supp}_{G}\left(M \otimes_{k} N\right)=\operatorname{supp}_{G}(M) \cap \operatorname{supp}_{G}(N)$.

(4) $\operatorname{cosupp}_{G} \operatorname{Hom}_{k}(M, N)=\operatorname{supp}_{G}(M) \cap \operatorname{cosupp}_{G}(N)$.

(5) $\operatorname{supp}_{G}(k)=\operatorname{Proj} H^{*}(G, k)=\operatorname{cosupp}_{G}(k)$.

Keeping in mind Theorem 2.7, parts (1) and (2) are recombinations of [Benson et al. 2016, Theorem 5.3 and Corollary 9.4]. Parts (3) and (4) are from [Benson et al. 2017, Theorem 4.4], while (5) is contained in [Benson et al. 2017, Lemma 4.5].

Remark 2.9. For an ideal $\mathfrak{a}$ in $H^{*}(G, k)$ we write $\mathscr{V}(\mathfrak{a})$ for the closed subset of those points in Proj $H^{*}(G, k)$ corresponding to homogeneous prime ideals containing $\mathfrak{a}$. 
Let $\zeta_{1}, \ldots, \zeta_{n}$ be a system of homogeneous generators of an ideal $\mathfrak{a} \subset H^{*}(G, k)$. By a theorem of Carlson [1983], one has $\operatorname{supp}_{G}(k / / \zeta)=\mathscr{V}(\zeta)$ for any $\zeta \in H^{d}(G, k)$. The tensor product property, recalled in Remark 2.8, now implies that

$$
\operatorname{supp}_{G}(k / / \mathfrak{a})=\mathscr{V}\left(\zeta_{1}\right) \cap \cdots \cap \mathscr{V}\left(\zeta_{n}\right)=\mathscr{V}(\mathfrak{a}) .
$$

In particular, for $L$ and $\mathfrak{q}$ as in Theorem 2.5 , one gets

$$
\operatorname{supp}_{G_{L}}(L / / \mathfrak{q})=\mathscr{V}(\mathfrak{q})=\{\sqrt{\mathfrak{q}}\} \subset \operatorname{Proj} H^{*}\left(G_{L}, L\right),
$$

since $\sqrt{\mathfrak{q}}$ is a closed point in $\operatorname{Proj} H^{*}\left(G_{L}, L\right)$.

\section{Point modules}

In this section we discuss a distinguished class of $G$-modules that correspond to a $\pi$ point. Later on we will see that these modules serve as cogenerators of colocalising subcategories.

Point modules. Fix a $\pi$-point $\alpha: k[t] /\left(t^{p}\right) \rightarrow k G$. The restriction functor

$$
\alpha^{*}: \operatorname{Mod} G \rightarrow \operatorname{Mod} k[t] /\left(t^{p}\right)
$$

admits a left adjoint and a right adjoint,

$$
\alpha_{*}:=k G \otimes_{k[t] /\left(t^{p}\right)}-\quad \text { and } \quad \alpha_{!}:=\operatorname{Hom}_{k[t] /\left(t^{p}\right)}(k G,-) .
$$

These functors are isomorphic, as the next result asserts.

Theorem 3.1. For any $\pi$-point $\alpha: k[t] /\left(t^{p}\right) \rightarrow k G$ and $k[t] /\left(t^{p}\right)$-module $M$, there is a natural isomorphism of $G$-modules

$$
\alpha_{*}(M) \cong \alpha_{!}(M) .
$$

Proof. It is convenient to set $R:=k[t] /\left(t^{p}\right)$. It is easy to verify that the $R$-module $\operatorname{Hom}_{k}(R, k)$ is isomorphic to $R$. This will be used further below. We will also use the fact that $k G$ is a Frobenius algebra, that is to say that there is an isomorphism of $G$-modules

$$
k G \cong \operatorname{Hom}_{k}(k G, k) .
$$

See [Jantzen 2003, Lemma I.8.7; Skowroński and Yamagata 2011, Chapter VI, Theorem 3.6]. This justifies the third step in the following chain of isomorphisms of $G$-modules:

$$
\operatorname{Hom}_{R}(k G, R) \cong \operatorname{Hom}_{R}\left(k G, \operatorname{Hom}_{k}(R, k)\right) \cong \operatorname{Hom}_{k}(k G, k) \cong k G .
$$

The second is standard adjunction.

We are now ready to justify the stated result. Consider first the case when $G$ is abelian. Then $k G$ and $\operatorname{Hom}_{R}(k G, R)$ also have $G^{\text {op }}$-actions. As $G$ is abelian, the isomorphism (3.2) is compatible with these structures. It follows that it is also 
compatible with the induced $R^{\mathrm{op}}$-actions on $k G$ and $\operatorname{Hom}_{R}(k G, R)$. This justifies the second isomorphism below:

$$
\alpha_{!}(M)=\operatorname{Hom}_{R}(k G, M) \cong \operatorname{Hom}_{R}(k G, R) \otimes_{R} M \cong k G \otimes_{R} M=\alpha_{*}(M) .
$$

The first isomorphism holds because $k G$ is a finitely generated projective $R$-module. The composition of the maps is the desired isomorphism.

Now let $G$ be an arbitrary finite group scheme. By definition, the $\pi$-point $\alpha$ factors as $R \stackrel{\beta}{\longrightarrow} k U \hookrightarrow k G$, where $U$ is an unipotent abelian subgroup scheme of $G$. Note that $\beta_{*}=\beta_{\text {! }}$ by what we have already verified, since $U$ is abelian. Observing that $\alpha_{*}=\operatorname{coind}_{U}^{G} \beta_{*}$ and $\alpha_{!}=\operatorname{ind}_{U}^{G} \beta_{!}$, it thus remains to show that $\operatorname{coind}_{U}^{G} \cong \operatorname{ind}_{U}^{G}$. By [Jantzen 2003, Proposition I.8.17], there is an isomorphism

$$
\operatorname{coind}_{U}^{G}(M) \cong \operatorname{ind}_{U}^{G}\left(M \otimes_{k}\left(\delta_{G}\right) \downarrow_{U} \delta_{U}^{-1}\right),
$$

where $\delta_{G}$ and $\delta_{U}$ are certain characters of $G$ and $U$, respectively. Since $U$ is a unipotent group scheme, it has no nontrivial characters; see [Waterhouse 1979, Section 8.3], for example. This yields the last claim and therefore the proof is complete.

Definition 3.3. Let $K$ be a field extension of $k$ and $\alpha: K[t] /\left(t^{p}\right) \rightarrow K G$ a $\pi$-point. We call the $G$-module

$$
\Delta_{G}(\alpha):=\operatorname{res}_{k}^{K} \alpha_{*}(K) \cong \operatorname{res}_{k}^{K} \alpha_{!}(K)
$$

the point module corresponding to $\alpha$.

As an example, we describe the point modules for the Klein four group, following the description of the $\pi$-points in [Friedlander and Pevtsova 2007, Example 2.3]; see also [Benson et al. 2017, Example 3.6].

Example 3.4. Let $V=\mathbb{Z} / 2 \times \mathbb{Z} / 2$ and $k$ a field of characteristic two. The group algebra $k V$ is isomorphic to $k[x, y] /\left(x^{2}, y^{2}\right)$, where $x+1$ and $y+1$ correspond to the generators of $V$, and Proj $H^{*}(V, k) \cong \mathbb{P}_{k}^{1}$. A $k V$-module $M$ is given by a $k$-vector space together with two $k$-linear endomorphisms $x_{M}$ and $y_{M}$, representing the action of $x$ and $y$, respectively.

For each closed point $\mathfrak{p} \in \mathbb{P}_{k}^{1}$ there is some finite field extension $K$ of $k$ such that $\mathbb{P}_{K}^{1}$ contains a rational point $[a, b]$ over $\mathfrak{p}$ (using homogeneous coordinates). The $\pi$-point corresponding to $\mathfrak{p}$ is represented by the map of $K$-algebras

$$
K[t] /\left(t^{p}\right) \rightarrow K[x, y] /\left(x^{2}, y^{2}\right), \quad \text { where } t \mapsto a x+b y,
$$

and the corresponding point module is given by $\Delta=K \oplus K$ together with

$$
x_{\Delta}=\left[\begin{array}{ll}
0 & 0 \\
b & 0
\end{array}\right] \text { and } y_{\Delta}=\left[\begin{array}{ll}
0 & 0 \\
a & 0
\end{array}\right] \text {. }
$$


Now let $K$ denote the field of rational functions in a variable $s$. The generic point of $\mathbb{P}_{k}^{1}$ then corresponds to the map of $K$-algebras

$$
K[t] /\left(t^{p}\right) \rightarrow K[x, y] /\left(x^{2}, y^{2}\right), \quad \text { where } t \mapsto x+s y,
$$

and the corresponding point module is given by $\Delta=K \oplus K$ together with

$$
x_{\Delta}=\left[\begin{array}{ll}
0 & 0 \\
s & 0
\end{array}\right] \text { and } y_{\Delta}=\left[\begin{array}{ll}
0 & 0 \\
1 & 0
\end{array}\right] .
$$

The next example illustrates that the $G$-module $\Delta_{G}(\alpha)$ depends on $\alpha$ and not only on the point in $\operatorname{Proj} H^{*}(G, k)$ that it represents.

Example 3.5. Let $k$ be a field of characteristic $p \geq 3$ and set $G:=\mathbb{Z} / p \times \mathbb{Z} / p$. Thus, $k G=k[x, y] /\left(x^{p}, y^{p}\right)$ and $\operatorname{Proj} H^{*}(G, k)=\mathbb{P}_{k}^{1}$. The homomorphism

$$
\alpha_{\lambda}: k[t] /\left(t^{p}\right) \rightarrow k G, \quad \text { where } t \mapsto x-\lambda y^{2},
$$

defines a $\pi$-point for any $\lambda \in k$, corresponding to the same point in $\mathbb{P}_{k}^{1}$, namely $[1,0]$. On the other hand, the point modules

$$
\Delta_{G}\left(\alpha_{\lambda}\right) \cong k[x, y] /\left(x-\lambda y^{2}, y^{p}\right)
$$

are pairwise nonisomorphic; for example, their annihilators differ. They are also indecomposable, because they are cyclic and $k G$ is a local ring.

The next example shows that point modules need not be indecomposable.

Example 3.6. Let $k$ be a field of characteristic 3 and set $G:=\Sigma_{3} \times \mathbb{Z} / 3$. The $\pi$ point $\alpha: k[t] /\left(t^{3}\right) \rightarrow k G$ given by the inclusion $\mathbb{Z} / 3 \hookrightarrow G$ as a direct factor yields a point module $\Delta_{G}(\alpha)$ that decomposes into two nonisomorphic indecomposable $G$-modules, because it is isomorphic to $k \Sigma_{3}$.

Endofinite modules. Let $G$ be a group scheme defined over $k$. A point module defined over a field extension $K$ is finite-dimensional, as a $G$-module, if and only if $K$ is finite-dimensional over $k$. Nonetheless, point modules always enjoy a strong finiteness property because they arise as restrictions of finite-dimensional modules.

Let $A$ be any ring. Following Crawley-Boevey [1991; 1992], we say that an $A$ module $M$ is endofinite if it has finite composition length when viewed as a module over its endomorphism ring $\operatorname{End}_{A}(M)$. The following result, due to CrawleyBoevey, collects some of the basic properties of endofinite modules. The proof employs the fact that endofinite modules are $\Sigma$-pure-injective.

Theorem 3.7. An indecomposable endofinite module has a local endomorphism ring and any endofinite module can be written essentially uniquely as a direct sum of indecomposable endofinite modules. Conversely, a direct sum of endofinite 
modules is endofinite if and only if there are only finitely many isomorphism classes of indecomposables involved.

The class of endofinite modules is closed under finite direct sums, direct summands, and arbitrary products or direct sum of copies of one module.

Proof. See [Crawley-Boevey 1991, Section 1.1; 1992, Section 4].

For an $A$-module $M$, we write $\operatorname{Add}(M)$ for the full subcategory of $A$-modules that are direct summands of direct sums of copies of $M$. Analogously, $\operatorname{Prod}(M)$ denotes the subcategory of all direct summands of products of copies of $M$. For an endofinite module $M$ it follows from Theorem 3.7 that $\operatorname{Add}(M)$ and $\operatorname{Prod}(M)$ coincide: they consist of all direct sums of indecomposable direct summands of $M$. This observation explains the formal part of the following proposition:

Proposition 3.8. For any $\pi$-point $\alpha$ of $G$, the $G$-module $\Delta_{G}(\alpha)$ is endofinite and there is an equality

$$
\operatorname{Add}\left(\Delta_{G}(\alpha)\right)=\operatorname{Prod}\left(\Delta_{G}(\alpha)\right) .
$$

Proof. Let $\alpha: K[t] /\left(t^{p}\right) \rightarrow K G$ be the given $\pi$-point. Then $\Delta_{G}(\alpha)$ is a $k G-K$ bimodule and there is a homomorphism of rings $K \rightarrow \operatorname{End}_{G}\left(\Delta_{G}(\alpha)\right)$. In particular, $\operatorname{dim}_{K}\left(\Delta_{G}(\alpha)\right)$ is an upper bound for the length of $\Delta_{G}(\alpha)$ as a module over $\operatorname{End}_{G}\left(\Delta_{G}(\alpha)\right)$. Since one has inequalities

$$
\operatorname{dim}_{K}\left(\Delta_{G}(\alpha)\right)=(1 / p) \operatorname{dim}_{K}(K G) \leq \operatorname{dim}_{K}(K G)<\infty,
$$

it follows that $\Delta_{G}(\alpha)$ is endofinite. The remaining assertion is by Theorem 3.7.

Support and cosupport. Next we explain how point modules can be used to compute support and cosupport; this is partly why we are interested in them.

Proposition 3.9. Let $\alpha$ be a $\pi$-point corresponding to $\mathfrak{p} \in \operatorname{Proj} H^{*}(G, k)$ and $M a$ $G$-module. The following statements are equivalent:

(1) $\mathfrak{p} \notin \operatorname{cosupp}_{G}(M)$.

(2) $\operatorname{Hom}_{k}\left(\Delta_{G}(\alpha), M\right)$ is projective.

(3) $\underline{\operatorname{Hom}}_{G}\left(\Delta_{G}(\alpha), M\right)=0$.

(4) $\underline{\operatorname{Hom}}_{G}^{*}\left(\Delta_{G}(\alpha), M\right)=0$.

Proof. The equivalences (1) $\Longleftrightarrow(2) \Longleftrightarrow(3)$ are [Benson et al. 2016, Lemma 9.2]. $(1) \Longleftrightarrow(4)$ : With $\alpha$ the map $K[t] /\left(t^{p}\right) \rightarrow K G$, adjunctions yield isomorphisms $\underline{\operatorname{Hom}}_{G}^{*}\left(\operatorname{res}_{k}^{K} \alpha_{*}(K), M\right) \cong \underline{\operatorname{Hom}}_{G_{K}}^{*}\left(\alpha_{*}(K), M^{K}\right) \cong \underline{\operatorname{Hom}}_{K[t] /\left(t^{p}\right)}^{*}\left(K, \alpha^{*}\left(M^{K}\right)\right)$.

Clearly, the right-hand term vanishes if and only if $\alpha^{*}\left(M^{K}\right)$ is projective.

Here is the analogous statement for supports. As in the context of commutative rings, one can use also tensor products with the point modules to detect support. 
Proposition 3.10. Let $\alpha$ be a $\pi$-point corresponding to $\mathfrak{p} \in \operatorname{Proj} H^{*}(G, k)$ and $M$ a $G$-module. The following statements are equivalent:

(1) $\mathfrak{p} \notin \operatorname{supp}_{G}(M)$.

(2) $\Delta_{G}(\alpha) \otimes_{k} M$ is projective.

(3) $\operatorname{Hom}_{k}\left(M, \Delta_{G}(\alpha)\right)$ is projective.

(4) $\underline{\operatorname{Hom}}_{G}\left(M, \Delta_{G}(\alpha)\right)=0$.

(5) $\underline{\operatorname{Hom}}_{G}^{*}\left(M, \Delta_{G}(\alpha)\right)=0$.

Proof. (1) $\Longleftrightarrow(2): \operatorname{Since} \operatorname{supp}_{G}\left(\Delta_{G}(\alpha)\right)=\{\mathfrak{p}\}$ by [Benson et al. 2016, Lemma 9.1], Remark 2.8(3) yields the first equivalence below:

$\mathfrak{p} \notin \operatorname{supp}_{G}(M) \Longleftrightarrow \operatorname{supp}_{G}\left(\Delta_{G}(\alpha) \otimes_{k} M\right)=\varnothing \Longleftrightarrow \Delta_{G}(\alpha) \otimes_{k} M$ is projective.

The second one holds because support detects projectivity, by Remark 2.8(1).

$(1) \Longleftrightarrow(4)$ : With $\alpha$ the map $K[t] /\left(t^{p}\right) \rightarrow K G$, adjunctions yield isomorphisms

$\underline{\operatorname{Hom}}_{G}\left(M, \operatorname{res}_{k}^{K} \alpha_{!}(K)\right) \cong \underline{\operatorname{Hom}}_{G_{K}}\left(M_{K}, \alpha_{!}(K)\right) \cong \underline{\operatorname{Hom}}_{K[t] /\left(t^{p}\right)}\left(\alpha^{*}\left(M_{K}\right), K\right)$.

Clearly, the right-hand term vanishes if and only if $\alpha^{*}\left(M_{K}\right)$ is projective.

$(1) \Longleftrightarrow(5)$ : This is analogous to (1) $\Longleftrightarrow(4)$.

$(1) \Longrightarrow(3)$ : When $\mathfrak{p}$ is not in $\operatorname{supp}_{G}(M)$, it is not in $\operatorname{supp}_{G}\left(C \otimes_{k} M\right)$ for any finite-dimensional $G$-module $C$, by Remark 2.8(3). Thus, the already-established equivalence of conditions (1) and (4) yields that

$$
\underline{\operatorname{Hom}}_{G}\left(C, \operatorname{Hom}_{k}\left(M, \Delta_{G}(\alpha)\right)\right) \cong \underline{\operatorname{Hom}}_{G}\left(C \otimes_{k} M, \Delta_{G}(\alpha)\right)=0 .
$$

Therefore $\operatorname{Hom}_{k}\left(M, \Delta_{G}(\alpha)\right)$ is projective.

(3) $\Longrightarrow(4)$ : This is clear.

In the next result, the claim about the support of $\Delta_{G}(\alpha)$ is from [Benson et al. 2016, Lemma 9.1], and has been used in the proofs of the Propositions 3.9 and 3.10.

Corollary 3.11. Let $\alpha$ be a $\pi$-point of $G$. $A \pi$-point $\beta$ of $G$ is equivalent to $\alpha$ if and only if $\underline{\operatorname{Hom}}_{G}^{*}\left(\Delta_{G}(\beta), \Delta_{G}(\alpha)\right) \neq 0$. In particular, there are equalities

$$
\operatorname{supp}_{G}\left(\Delta_{G}(\alpha)\right)=\{\mathfrak{p}\}=\operatorname{cosupp}_{G}\left(\Delta_{G}(\alpha)\right),
$$

where $\mathfrak{p}$ is the point in Proj $H^{*}(G, k)$ corresponding to $\alpha$.

Proof. If $\beta$ corresponds to a point $\mathfrak{q}$ in Proj $H^{*}(G, k)$, then $\operatorname{supp}_{G}\left(\Delta_{G}(\beta)\right)=\{\mathfrak{q}\}$ by [loc. cit.], so Proposition 3.10 yields that $\underline{\operatorname{Hom}}_{G}^{*}\left(\Delta_{G}(\beta), \Delta_{G}(\alpha)\right)$ is nonzero precisely when $\mathfrak{q}=\mathfrak{p}$. Given this, it follows from Proposition 3.9 that the cosupport of $\Delta_{G}(\alpha)$ is $\{\mathfrak{p}\}$. 


\section{4. $\mathfrak{p}$-local and $\mathfrak{p}$-complete objects}

The proof of Theorem 1.1 amounts to showing that for any homogeneous prime ideal $\mathfrak{p}$ of $H^{*}(G, k)$ the $\mathfrak{p}$-local and $\mathfrak{p}$-complete objects in StMod $G$ form a minimal Hom closed colocalising subcategory. Here, a Hom closed colocalising subcategory $C \subseteq$ StMod $G$ is minimal if $C^{\prime} \subseteq C$ implies $C^{\prime}=0$ or $C^{\prime}=C$ for any Hom closed colocalising subcategory $\mathrm{C}^{\prime} \subseteq \operatorname{StMod} G$.

$\mathfrak{p}$-local and $\mathfrak{p}$-complete objects. We recall from [Benson et al. 2008; 2012] the definitions and basic facts about $\mathfrak{p}$-local and $\mathfrak{p}$-complete objects in StMod $G$.

Fix $\mathfrak{p} \in \operatorname{Proj} H^{*}(G, k)$. We write $\Gamma_{\mathfrak{p}} \operatorname{StMod} G$ for the full subcategory of $G$ modules $M$ such that $\Gamma_{\mathfrak{p}}(M) \cong M$ and have, from Corollary 5.9 of [Benson et al. 2008],

$$
\Gamma_{\mathfrak{p}} \operatorname{StMod} G=\left\{M \in \operatorname{StMod} G \mid \operatorname{supp}_{G}(M) \subseteq\{\mathfrak{p}\}\right\} .
$$

From [Benson et al. 2012, Corollaries 4.8 and 4.9], it follows that a $G$-module $M$ satisfies $\Lambda^{\mathfrak{p}}(M) \cong M$ if and only if $M$ is $\mathfrak{p}$-local and $\mathfrak{p}$-complete, and that

$$
\Lambda^{\mathfrak{p}} \operatorname{StMod} G=\left\{M \in \operatorname{StMod} G \mid \operatorname{cosupp}_{G}(M) \subseteq\{\mathfrak{p}\}\right\} .
$$

Note that the adjoint pair $\left(\Gamma_{\mathfrak{p}}, \Lambda^{\mathfrak{p}}\right)$ restricts by [Benson et al. 2012, Proposition 5.1] to an equivalence

$$
\Gamma_{\mathfrak{p}} \operatorname{StMod} G \stackrel{\sim}{\longrightarrow} \Lambda^{\mathfrak{p}} \operatorname{StMod} G .
$$

Cogenerators for $\mathfrak{p}$-local and $\mathfrak{p}$-complete objects. Given a set $T$ of $G$-modules, let $\operatorname{Coloc}(T)$ be the smallest colocalising subcategory of StMod $G$ that contains $T$. We say that $T$ cogenerates a class $C$ of $G$-modules if $C \subseteq \operatorname{Coloc}(T)$. The class C is Hom closed if, for every pair of $G$-modules $M$ and $N$ with $N \in \mathrm{C}$, we have $\operatorname{Hom}_{k}(M, N) \in \mathrm{C}$. We write Coloc ${ }^{\mathrm{Hom}}(T)$ for the smallest Hom closed colocalising subcategory that contains $T$.

An object $T$ is a perfect cogenerator of a colocalising subcategory $\mathrm{C} \subseteq \mathrm{StMod} G$ if the following holds:

(1) If $M$ is an object in $\mathrm{C}$ and $\underline{\operatorname{Hom}}_{G}(M, T)=0$ then $M=0$.

(2) If a countable family of morphisms $M_{i} \rightarrow N_{i}$ in $\mathrm{C}$ is such that, for all $i$,

$$
\underline{\operatorname{Hom}}_{G}\left(N_{i}, T\right) \rightarrow \underline{\operatorname{Hom}}_{G}\left(M_{i}, T\right)
$$

is surjective, then so is the induced map

$$
\underline{\operatorname{Hom}}_{G}\left(\prod_{i} N_{i}, T\right) \rightarrow \underline{\operatorname{Hom}}_{G}\left(\prod_{i} M_{i}, T\right) .
$$

Any perfect cogenerator is a cogenerator; see [Benson et al. 2012, Section 5].

Recall from Remark 2.4 that any closed point of Proj $H^{*}(G, k)$ is represented by a $\pi$-point $\alpha: K[t] / t^{p} \rightarrow K G$ defined over a finite field extension $K / k$. 
Lemma 4.1. Let $\alpha: K[t] /\left(t^{p}\right) \rightarrow K G$ be a $\pi$-point representing $\mathfrak{p} \in \operatorname{Proj} H^{*}(G, k)$. If $K$ is finite-dimensional over $k$, then $\Delta_{G}(\alpha)$ perfectly cogenerates $\Lambda^{\mathfrak{p}} \operatorname{StMod} G$. Proof. We check the conditions (1) and (2) for $\Delta_{G}(\alpha)$.

(1) If $M \in \Lambda^{\mathfrak{p}}$ StMod $G$ is nonzero, then $\operatorname{cosupp}_{G}(M)=\{\mathfrak{p}\}$ and hence $\mathfrak{p}$ is in $\operatorname{supp}_{G}(M)$ by Remark 2.8(2). Thus, $\underline{\operatorname{Hom}}_{G}\left(M, \Delta_{G}(\alpha)\right) \neq 0$ by Proposition 3.10.

(2) Since extension of scalars is left adjoint to restriction of scalars, we have

$$
\underline{\operatorname{Hom}}_{G}\left(M, \Delta_{G}(\alpha)\right) \cong \underline{\operatorname{Hom}}_{G_{K}}\left(M_{K}, \alpha_{*}(K)\right) .
$$

As $\alpha_{*}(K)$ is finite-dimensional as a $G_{K}$-module, using the duality isomorphism (2.1) we may rewrite the right-hand term as

$$
\underline{\operatorname{Hom}}_{G_{K}}\left(\tau^{-1} \Omega\left(\alpha_{*}(K)\right), M_{K}\right)^{\vee} .
$$

So $\underline{\operatorname{Hom}}_{G}\left(N, \Delta_{G}(\alpha)\right) \rightarrow \underline{\operatorname{Hom}}_{G}\left(M, \Delta_{G}(\alpha)\right)$ is surjective if and only if

$$
\underline{\operatorname{Hom}}_{G_{K}}\left(\tau^{-1} \Omega\left(\alpha_{*}(K)\right), M_{K}\right) \rightarrow \underline{\operatorname{Hom}}_{G_{K}}\left(\tau^{-1} \Omega\left(\alpha_{*}(K)\right), N_{K}\right)
$$

is injective. It remains to observe that $M \mapsto M_{K}$ preserves products, as $K$ is finitedimensional over $k$.

Let $I$ be an injective $H^{*}(G, k)$-module and $C$ a finite-dimensional $G$-module. In what follows, we use the representing objects $T_{C}(I)$ and the Koszul objects $k / / \mathfrak{p}$ defined in Section 1.

Lemma 4.2. Fix a point $\mathfrak{p}$ in $\operatorname{Proj} H^{*}(G, k)$ and $I$ an injective $H^{*}(G, k)$-module.

(1) For any finite-dimensional $G$-modules $C$ and $M$, there is a natural isomorphism

$$
\operatorname{Hom}_{k}\left(M, T_{C}(I)\right) \cong T_{\mathrm{Hom}_{k}(M, C)}(I) .
$$

(2) With I the injective envelope of $H^{*}(G, k) / \mathfrak{p}$, the modules $\operatorname{Hom}_{k}\left(k / / \mathfrak{p}, T_{C}(I)\right)$, as $C$ varies over the simple $G$-modules, perfectly cogenerate $\Lambda^{\mathfrak{p}} \mathrm{StMod} G$.

Proof. Recall that $(-)^{\vee}$ denotes the functor $\operatorname{Hom}_{k}(-, k)$. For a $G$-module $M$, we consider $M^{\vee}$ with the diagonal $G$-action, and we have

$$
\operatorname{Hom}_{k}(M,-) \cong-\otimes_{k} M^{\vee}
$$

when $M$ is finite-dimensional. Combining this with standard adjunctions and the definition of $T_{C}(I)$ gives the following isomorphisms, which justify (1):

$$
\begin{aligned}
\underline{\operatorname{Hom}}_{G}\left(-, \operatorname{Hom}_{k}\left(M, T_{C}(I)\right)\right) & \cong \underline{\operatorname{Hom}}_{G}\left(-\otimes_{k} M, T_{C}(I)\right) \\
& \cong \operatorname{Hom}_{H^{*}(G, k)}\left(\underline{\operatorname{Hom}}_{G}^{*}\left(C,-\otimes_{k} M\right), I\right) \\
& \cong \operatorname{Hom}_{H^{*}(G, k)}\left(\underline{\operatorname{Hom}}_{G}^{*}\left(C \otimes_{k} M^{\vee},-\right), I\right) \\
& \cong \operatorname{Hom}_{H^{*}(G, k)}\left(\underline{\operatorname{Hom}}_{G}^{*}\left(\operatorname{Hom}_{k}(M, C),-\right), I\right) \\
& \cong \underline{\operatorname{Hom}}_{G}\left(-, T_{\operatorname{Hom}_{k}(M, C)}(I)\right) .
\end{aligned}
$$


As to (2), given the isomorphism in (1) applied to $M=k / / \mathfrak{p}$, one can deduce the desired result by mimicking the proof of [Benson et al. 2012, Proposition 5.4].

For the next result we employ the reduction to closed points technique from Section 1.

Proposition 4.3. Let $\mathfrak{p}$ be a point in Proj $H^{*}(G, k)$ and $M$ a $\mathfrak{p}$-local $G$-module. There exists a field extension $L / k$ and an ideal $\mathfrak{q}$ in $H^{*}\left(G_{L}, L\right)$ with radical a closed point in Proj $H^{*}\left(G_{L}, L\right)$ lying over $\mathfrak{p}$ such that $\operatorname{res}_{k}^{L} \operatorname{Hom}_{L}\left(L / / \mathfrak{q}, M^{L}\right)$ and $\operatorname{Hom}_{k}(k / / \mathfrak{p}, M)$ are isomorphic as $G$-modules.

Proof. By Theorem 2.5, we can find $L$ and $\mathfrak{q}$ such that there is an isomorphism $\operatorname{res}_{k}^{L}(L / / \mathfrak{q}) \cong(k / / \mathfrak{p})_{\mathfrak{p}}$. Thus there are isomorphisms

$$
\begin{aligned}
\operatorname{res}_{k}^{L} \operatorname{Hom}_{L}\left(L / / \mathfrak{q}, M^{L}\right) & \cong \operatorname{Hom}_{k}\left(\operatorname{res}_{k}^{L}(L / / \mathfrak{q}), M\right) \\
& \cong \operatorname{Hom}_{k}\left((k / / \mathfrak{p})_{\mathfrak{p}}, M\right) \\
& \cong \operatorname{Hom}_{k}(k / / \mathfrak{p}, M) .
\end{aligned}
$$

The first one follows from Lemma 2.2 and the last one holds as $M$ is $\mathfrak{p}$-local.

In what follows, Thick $(M)$ denotes the thick subcategory of StMod $G$ generated by a $G$-module $M$.

Theorem 4.4. Given $\mathfrak{p} \in \operatorname{Proj} H^{*}(G, k)$, there exists a $\pi$-point $\alpha: K[t] /\left(t^{p}\right) \rightarrow K G$ corresponding to $\mathfrak{p}$ that factors through a quasielementary subgroup scheme of $G_{K}$ and has the following properties:

(1) $\Delta_{G}(\alpha)$ is a compact object in (StMod $\left.G\right)_{\mathfrak{p}}$.

(2) $\operatorname{Coloc}\left(\Delta_{G}(\alpha)\right)=\Lambda^{\mathfrak{p}} \operatorname{StMod} G$.

Proof. Let $L$ and $\mathfrak{q}$ be as in Proposition 4.3, and let $\mathfrak{m}=\sqrt{\mathfrak{q}}$. Since $\mathfrak{m}$ is a closed point in Proj $H^{*}\left(G_{L}, L\right)$, there exists a finite extension $K$ of $L$ and a $\pi$ point $\alpha: K[t] /\left(t^{p}\right) \rightarrow K G$ of $G_{L}$ corresponding to $\mathfrak{m}$, and factoring through a quasielementary subgroup scheme of $G_{K}$; see Remark 2.4. It then follows directly from the definitions that $\alpha$ corresponds to $\mathfrak{p}$, when viewed as a $\pi$-point of $G$.

(1) Set $M:=L / / \mathfrak{q}$. This is a finite-dimensional $G_{L}$-module with support $\{\mathfrak{m}\}$; see Remark 2.9. From the construction it is clear that the $G_{L}$-module $\operatorname{res}_{L}^{K} \alpha_{*}(K)$ is also finite-dimensional and has support $\{\mathfrak{m}\}$. Thus the classification [Benson et al. 2016, Corollary 10.2] of tensor closed thick subcategories of stmod $G_{L}$ yields that $\operatorname{res}_{L}^{K} \alpha_{*}(K)$ is in Thick ${ }^{\otimes}(M)$. Any simple $G_{L}$-module is a direct summand of $S_{L}$, where $S$ is the sum of representatives of isomorphism classes of simple $G$-modules, so one gets

$$
\operatorname{res}_{L}^{K} \alpha_{*}(K) \in \operatorname{Thick}\left(M \otimes_{L} S_{L}\right) \text {. }
$$


Applying $\operatorname{res}_{k}^{L}$ and using Lemma 2.2, one then gets that

$$
\Delta_{G}(\alpha)=\operatorname{res}_{k}^{L} \operatorname{res}_{L}^{K} \alpha_{*}(K) \in \operatorname{Thick}\left(\left(\operatorname{res}_{k}^{L} M\right) \otimes_{k} S\right) .
$$

It remains to verify that $\left(\operatorname{res}_{k}^{L} M\right) \otimes_{k} S$ is a compact object in (StMod $\left.G\right)_{\mathfrak{p}}$. To this end, note that there are isomorphisms

$$
\left(\operatorname{res}_{k}^{L} M\right) \otimes_{k} S \cong(k / / \mathfrak{p})_{\mathfrak{p}} \otimes_{k} S \cong\left(k / / \mathfrak{p} \otimes_{k} S\right)_{\mathfrak{p}},
$$

where the first one is by Theorem 2.5 and the second is by [Benson et al. 2008, Theorem 8.2], for example. It remains to note that $k / / \mathfrak{p} \otimes_{k} S$ is a finite-dimensional $G$-module and hence compact in $\operatorname{StMod} G$, so that its localisation at $\mathfrak{p}$ is compact in $(\operatorname{StMod} G)_{\mathfrak{p}}$.

(2) Let $I$ denote the injective envelope of the $H^{*}(G, k)$-module $H^{*}(G, k) / \mathfrak{p}$. Since $\operatorname{supp}_{G_{L}}(L / / \mathfrak{q})=\{\mathfrak{m}\}$, Remark 2.8(4) implies that for any finite-dimensional $G$ module $C$ the module $\operatorname{Hom}_{L}\left(L / / \mathfrak{q}, T_{C}(I)^{L}\right)$ belongs to $\Lambda^{\mathfrak{m}} \operatorname{StMod} G_{L}$. Given the choice of $\alpha$, Lemma 4.1 thus implies that this module is cogenerated by $\Delta_{G_{L}}(\alpha)$. So, by Proposition 4.3 , the $G$-module $\operatorname{res}_{k}^{L} \Delta_{G_{L}}(\alpha)$, that is to say $\Delta_{G}(\alpha)$, cogenerates $\operatorname{Hom}_{k}\left(k / / \mathfrak{p}, T_{C}(I)\right)$. It remains to apply Lemma 4.2(2).

Minimality. Next we prove that $\Lambda^{\mathfrak{p}}$ StMod $G$ is a minimal Hom closed colocalising subcategory. This requires further preparation.

Lemma 4.5. Let $K$ be a field extension of $k$ and $H$ a subgroup scheme of $G_{K}$. Set $F=\operatorname{res}_{k}^{K} \operatorname{coind}_{H}^{G_{K}}(K)$. If $M$ is a $G$-module then

$$
\operatorname{res}_{k}^{K} \operatorname{ind}_{H}^{G_{K}} \operatorname{res}_{H}^{G_{K}}\left(M^{K}\right)=\operatorname{Hom}_{k}(F, M) .
$$

When $K$ is a finite extension of $k$, the $G$-module $F$ is finite-dimensional over $k$.

Proof. The desired result is a consequence of the isomorphisms

$$
\begin{aligned}
\operatorname{res}_{k}^{K} \operatorname{ind}_{H}^{G_{K}} \operatorname{res}_{H}^{G_{K}}\left(M^{K}\right) & \cong \operatorname{res}_{k}^{K} \operatorname{Hom}_{K}\left(\operatorname{coind}_{H}^{G_{K}}(K), M^{K}\right) \\
& \cong \operatorname{Hom}_{k}\left(\operatorname{res}_{k}^{K} \operatorname{coind}_{H}^{G_{K}}(K), M\right) .
\end{aligned}
$$

The first one follows from Lemma 2.3 and the second from Lemma 2.2. The last assertion follows from the fact that, in general, there are inequalities

$$
\operatorname{dim}_{K} \operatorname{coind}_{H}^{G_{K}}(K)=\frac{\operatorname{dim}_{K}(K G)}{\operatorname{dim}_{K}(K H)} \leq \operatorname{dim}_{K}(K G),
$$

and hence the number on the left is finite.

Lemma 4.6. Given a quasielementary group scheme $\mathscr{E}$ over $K$ and a $\pi$-point $\beta: K[t] /\left(t^{p}\right) \rightarrow K_{\mathscr{E}}^{\mathscr{E}}$, for any $\mathscr{E}$-module $M$ the $\mathscr{E}$-module $\beta_{!} \beta^{*}(M)$ is in Thick $(M)$. 


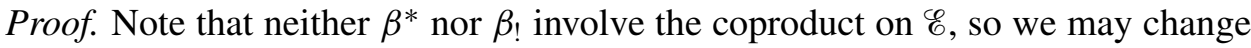
that and assume that $K U$ is the group algebra of an elementary abelian $p$-group and that $\beta$ is the inclusion $K H \rightarrow K_{\mathscr{E}}^{\mathscr{E}}$, where $H$ is a cyclic subgroup $\mathscr{E}$. Lemma 4.5 then yields that $\operatorname{ind}_{H}^{\mathscr{E}} \operatorname{res}_{H}^{\mathscr{E}}(M)$, that is to say $\beta_{!} \beta^{*}(M)$, equals $\operatorname{Hom}_{k}(F, M)$ for some finite-dimensional $\mathscr{E}$-module $F$. Since $k$ is the only simple $\mathscr{E}$-module, $F$ is in $\operatorname{Thick}(k)$, and hence $\operatorname{Hom}_{k}(F, M)$ is in $\operatorname{Thick}\left(\operatorname{Hom}_{k}(k, M)\right)$. It remains to recall that $\operatorname{Hom}_{k}(k, M) \cong M$ as $\mathscr{E}$-modules.

Combining the preceding results one obtains the following:

Proposition 4.7. Let $\alpha: K[t] /\left(t^{p}\right) \rightarrow K G$ be a $\pi$-point of $G$ that factors through a quasielementary subgroup scheme of $G_{K}$. Then $\operatorname{res}_{k}^{K} \alpha ! \alpha^{*}\left(M^{K}\right)$ is in $\operatorname{Coloc}^{\operatorname{Hom}}(M)$ for any $G$-module $M$.

Proof. By hypothesis, there exists a quasielementary subgroup scheme $U$ of $G_{K}$ such that $\alpha=\gamma \circ \beta$, where $\beta: K[t] /\left(t^{p}\right) \rightarrow K U$ and $\gamma: K U \rightarrow K G$. Then

$$
\operatorname{res}_{k}^{K} \alpha ! \alpha^{*}\left(M^{K}\right)=\operatorname{res}_{k}^{K} \gamma ! \beta_{!} \beta^{*} \gamma^{*}\left(M^{K}\right) .
$$

Since $\beta_{!} \beta^{*} \gamma^{*}\left(M^{K}\right)$ is in Thick $\left(\gamma^{*}\left(M^{K}\right)\right)$ by Lemma 4.6, one has that

$$
\operatorname{res}_{k}^{K} \alpha ! \alpha^{*}\left(M^{K}\right) \in \operatorname{Thick}\left(\operatorname{res}_{k}^{K} \gamma ! \gamma^{*}\left(M^{K}\right)\right) \text {. }
$$

Since $\operatorname{res}_{k}^{K} \gamma ! \gamma^{*}\left(M^{K}\right)$ is in $\operatorname{Coloc}^{\text {Hom }}(M)$ by Lemma 4.5, it follows that

$$
\operatorname{res}_{k}^{K} \alpha ! \alpha^{*}\left(M^{K}\right) \in \operatorname{Coloc}^{\mathrm{Hom}}(M) \text {. }
$$

The next result complements Theorem 4.4.

Theorem 4.8. Let $M$ be a $G$-module and $\mathfrak{p} \in \operatorname{cosupp}_{G}(M)$. If $\alpha: K[t] /\left(t^{p}\right) \rightarrow K G$ is a $\pi$-point that factors through a quasielementary subgroup scheme of $G_{K}$ and represents $\mathfrak{p}$, then $\Delta_{G}(\alpha)$ is in $\operatorname{Coloc}^{\mathrm{Hom}}(M)$.

Proof. By hypothesis on $\mathfrak{p}$, the $k[t] /\left(t^{p}\right)$-module $\alpha^{*}\left(M^{K}\right)$ is not projective, and hence $K$ is in $\operatorname{Coloc}\left(\alpha^{*}\left(M^{K}\right)\right)$. This implies that $\alpha_{!}(K)$ is in $\operatorname{Coloc}\left(\alpha_{!} \alpha^{*}\left(M^{K}\right)\right)$, and hence, by restriction of scalars, that

$$
\Delta_{G}(\alpha) \in \operatorname{Coloc}\left(\operatorname{res}_{k}^{K} \alpha ! \alpha^{*}\left(M^{K}\right)\right) .
$$

Finally, by Proposition 4.7, the module on the right is in $\operatorname{Coloc}^{\mathrm{Hom}}(M)$.

Corollary 4.9. For $\mathfrak{p} \in \operatorname{Proj} H^{*}(G, k)$, the colocalising subcategory $\Lambda^{\mathfrak{p}}$ StMod $G$ of StMod $G$ contains no proper nonzero Hom closed colocalising subcategories.

Proof. Fix a $\pi$-point $\alpha$ as in Theorem 4.4, factoring through a quasielementary subgroup scheme. Since $\mathfrak{p}$ is in the $\pi$-cosupport of any nonzero module $M$ in $\Lambda^{\mathfrak{p}}$ StMod $G$, Theorem 4.8 yields the inclusion below:

$$
\Lambda^{\mathfrak{p}} \operatorname{StMod} G=\operatorname{Coloc}\left(\Delta_{G}(\alpha)\right) \subseteq \operatorname{Coloc}^{\operatorname{Hom}}(M) .
$$

The equality is from Theorem 4.4. This is the desired result. 
Proof of Theorem 1.1. In the terminology of [Benson et al. 2012], Corollary 4.9 means that StMod $G$ is costratified by the action of $H^{*}(G, k)$. Given this, [Benson et al. 2012, Corollary 9.2] yields the desired bijection between Hom closed colocalising subcategories of StMod $G$ and subsets of Proj $H^{*}(G, k)$.

Colocalising and localising subcategories. A key step in the proof of the classification theorem above is that, given a point $\mathfrak{p}$ in $\operatorname{Proj} H^{*}(G, k)$, the point module associated to a certain type of $\pi$-point representing $\mathfrak{p}$ cogenerates $\Lambda^{\mathfrak{p}} \operatorname{StMod} G$; see Theorem 4.4. As a corollary of the classification result, it follows that any $\pi$ point may be used, as long as we also allow tensor products with simple modules.

Corollary 4.10. For any point $\mathfrak{p}$ in $\operatorname{Proj} H^{*}(G, k)$ and any $\pi$-point representing $\mathfrak{p}$,

$\operatorname{Loc}^{\otimes}\left(\Delta_{G}(\alpha)\right)=\Gamma_{\mathfrak{p}} \operatorname{StMod} G$ and $\operatorname{Coloc}^{\operatorname{Hom}}\left(\Delta_{G}(\alpha)\right)=\Lambda^{\mathfrak{p}} \operatorname{StMod} G$.

Proof. Since $\operatorname{supp}_{G}\left(\Delta_{G}(\alpha)\right)=\{\mathfrak{p}\}$, the first equality is a direct consequence of the bijection between tensor closed localising subcategories of StMod $G$ and subsets of Proj $H^{*}(G, k)$ established in [Benson et al. 2016, Theorem 8.1]. In the same vein, the second equality follows from Theorem 1.1, since $\operatorname{cosupp}_{G}\left(\Delta_{G}(\alpha)\right)=\{\mathfrak{p}\}$.

Given a subcategory C of StMod $G$ we set

$$
\operatorname{supp}_{G}(\mathrm{C}):=\bigcup_{M \in C} \operatorname{supp}_{G}(M) \text { and } \operatorname{cosupp}_{G}(\mathrm{C}):=\bigcup_{M \in \mathrm{C}} \operatorname{cosupp}_{G}(M) \text {. }
$$

For any subset $U \subseteq \operatorname{Proj} H^{*}(G, k)$ set

$$
\operatorname{cl}(\mathcal{U}):=\left\{\mathfrak{p} \in \operatorname{Proj} H^{*}(G, k) \mid \mathfrak{p} \subseteq \mathfrak{q} \text { for some } \mathfrak{q} \in \mathcal{U}\right\} .
$$

This is the closure of $U$ with respect to the Hochster dual of the Zariski topology [Hochster 1969], and we call $U$ generalisation closed if $\mathrm{cl}(U)=U$.

Corollary 4.11. For a subcategory $\mathrm{C} \subseteq$ StMod $G$ the following are equivalent:

(1) C is a tensor closed localising subcategory and closed under all products.

(2) C is a Hom closed colocalising subcategory and closed under all coproducts.

In that case we have $\operatorname{supp}_{G}(\mathrm{C})=\operatorname{cosupp}_{G}(\mathrm{C})$ and this set is generalisation closed Moreover, any generalisation closed subset of Proj $H^{*}(G, k)$ arises in that way.

Proof. Benson et al. [2016] prove that, as a tensor triangulated category, StMod $G$ is stratified by $H^{*}(G, k)$. It follows that the assignment $C \mapsto \operatorname{supp}_{G}(\mathrm{C})$ yields a bijection between the tensor closed localising subcategories of StMod $G$ that are closed under all products and the generalisation closed subsets of Proj $H^{*}(G, k)$. This can be verified by mimicking the argument used to prove the implication (a) $\Longleftrightarrow$ (c) of [Benson et al. 2011, Theorem 11.8]; see also [Benson et al. 2011, 
Theorem 6.3]. The desired assertion now follows from the bijection between localising and colocalising subcategories (Corollary 1.2), noticing that for any tensor ideal localising subcategory $\mathrm{C}$ we have

$$
\operatorname{supp}_{G}(\mathrm{C}) \sqcup \operatorname{cosupp}_{G}\left(\mathrm{C}^{\perp}\right)=\operatorname{Proj} H^{*}(G, k) .
$$

For any generalisation closed subset $U \subseteq \operatorname{Proj} H^{*}(G, k)$ we set

$$
(\operatorname{StMod} G)_{u}:=\left\{M \in \operatorname{StMod} G \mid \operatorname{supp}_{G}(M) \subseteq U\right\} .
$$

We collect some basic properties of this category.

Remark 4.12. There is an equality

$$
(\operatorname{StMod} G)_{U}=\left\{M \in \operatorname{StMod} G \mid \operatorname{cosupp}_{G}(M) \subseteq \mathcal{U}\right\}
$$

and this is compactly generated as a triangulated category. The first assertion is justified by Remark 2.8(2), and compact generation follows from the fact that

$$
(\operatorname{StMod} G)_{U}=\Gamma_{U^{c}}\left(\operatorname{StMod}_{G}\right)^{\perp},
$$

where $U^{c}:=\operatorname{Proj} H^{*}(G, k) \backslash U$. Indeed, the subset $U^{c}$ is specialisation closed, so $\Gamma_{\Upsilon^{c}}\left(\operatorname{StMod}_{G}\right)$ is compactly generated (see [Benson et al. 2011, Proposition 2.7], for example). Now the assertion is a formal consequence of [Neeman 1992, Theorem 2.1; 2001, Theorem 9.1.16].

Given generalisation closed subsets $\mathscr{V} \subseteq \mathcal{U} \subseteq \operatorname{Proj} H^{*}(G, k)$, it follows from Brown representability [Neeman 2001] that the inclusion

$$
(\operatorname{StMod} G)_{\mathscr{V}} \rightarrow(\operatorname{StMod} G)_{\varkappa}
$$

admits a left adjoint and a right adjoint, because the functor preserves products and coproducts.

Now fix a point $\mathfrak{p}$ in Proj $H^{*}(G, k)$ and consider the generalisation closure of $\mathfrak{p}$. Then $(\operatorname{StMod} G)_{\leq \mathfrak{p}}$ equals the full subcategory of $\mathfrak{p}$-local $G$-modules and we obtain the following pair of equivalent recollements:

$$
\begin{aligned}
& (\operatorname{StMod} G)_{<\mathfrak{p}} \stackrel{\longleftrightarrow}{\longleftarrow}(\operatorname{StMod} G)_{\leq \mathfrak{p}} \stackrel{\frac{\text { incl }}{\longleftarrow} \Gamma_{\mathfrak{p}} \longrightarrow}{\longleftarrow} \Gamma_{\mathfrak{p}}(\operatorname{StMod} G), \\
& (\operatorname{StMod} G)_{<\mathfrak{p}} \varlimsup_{\nVdash}^{\longleftarrow}(\operatorname{StMod} G)_{\leq \mathfrak{p}} \frac{\Gamma_{\mathfrak{p}}}{\longleftarrow \Lambda^{\mathfrak{p}} \longrightarrow} \Lambda^{\mathfrak{i n c l}}(\operatorname{StMod} G)
\end{aligned}
$$

Note that for a $\pi$-point $\alpha$ representing $\mathfrak{p}$ we have, in $(\operatorname{StMod} G)_{\leq \mathfrak{p}}$,

$$
\Delta_{G}(\alpha)^{\perp}=(\operatorname{StMod} G)_{<\mathfrak{p}}={ }^{\perp} \Delta_{G}(\alpha) .
$$

There is an analogy between point modules over finite group schemes and standard objects of highest weight categories. In fact, the analogy includes costandard 
objects, depending on whether one thinks of a point module as induced or coinduced from a trivial representation; see Theorem 3.1.

Remark 4.13. Let A be a highest weight category [Cline et al. 1988] with partially ordered set of weights $\Lambda$, which is assumed to be finite for simplicity. Thus $A$ is an abelian length category with simple objects $\{L(\lambda)\}_{\lambda \in \Lambda}$. Now fix $\lambda \in \Lambda$ and consider the full subcategory $A_{\leq \lambda}$ of objects in $A$ that have composition factors $L(\mu)$ with $\mu \leq \lambda$. The standard object $\Delta(\lambda)$ is a projective cover of $L(\lambda)$ in $\mathrm{A}_{\leq \lambda}$ and its endomorphism ring is a division ring, which we denote by $K_{\lambda}$. This situation gives rise to the following recollement [Cline et al. 1988, Theorem 3.9]:

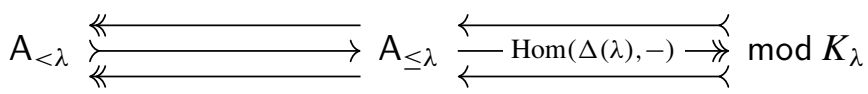

Note that $\Delta(\lambda)^{\perp}=\mathrm{A}_{<\lambda}={ }^{\perp} \nabla(\lambda)$, where $\nabla(\lambda)$ denotes the costandard object corresponding to $\lambda$, namely the injective envelope of $L(\lambda)$ in $\mathrm{A}_{\leq \lambda}$.

\section{References}

[Auslander 1978] M. Auslander, "Functors and morphisms determined by objects", pp. 1-244 in Representation theory of algebras (Philadelphia, 1976), edited by R. Gordon, Lecture Notes in Pure Appl. Math. 37, Dekker, New York, 1978. MR Zbl

[Benson and Krause 2002] D. Benson and H. Krause, "Pure injectives and the spectrum of the cohomology ring of a finite group", J. Reine Angew. Math. 542 (2002), 23-51. MR Zbl

[Benson et al. 2008] D. Benson, S. B. Iyengar, and H. Krause, "Local cohomology and support for triangulated categories”, Ann. Sci. Éc. Norm. Supér. (4) 41:4 (2008), 575-621. MR Zbl

[Benson et al. 2011] D. Benson, S. B. Iyengar, and H. Krause, "Stratifying triangulated categories", J. Topol. 4:3 (2011), 641-666. MR Zbl

[Benson et al. 2012] D. J. Benson, S. B. Iyengar, and H. Krause, "Colocalizing subcategories and cosupport", J. Reine Angew. Math. 673 (2012), 161-207. MR Zbl

[Benson et al. 2016] D. Benson, S. B. Iyengar, H. Krause, and J. Pevtsova, "Stratification for module categories of finite group schemes", preprint, 2016. arXiv

[Benson et al. 2017] D. Benson, S. B. Iyengar, H. Krause, and J. Pevtsova, "Stratification and $\pi$ cosupport: finite groups", Math. Z. (online publication February 2017).

[Brown 1965] E. H. Brown, Jr., "Abstract homotopy theory", Trans. Amer. Math. Soc. 119 (1965), 79-85. MR Zbl

[Carlson 1983] J. F. Carlson, "The varieties and the cohomology ring of a module", J. Algebra 85:1 (1983), 104-143. MR Zbl

[Carlson 1996] J. F. Carlson, Modules and group algebras, Birkhäuser, Basel, 1996. MR Zbl

[Cartan and Eilenberg 1956] H. Cartan and S. Eilenberg, Homological algebra, Princeton Univ. Press, 1956. MR Zbl

[Cline et al. 1988] E. Cline, B. Parshall, and L. Scott, "Finite-dimensional algebras and highest weight categories", J. Reine Angew. Math. 391 (1988), 85-99. MR Zbl

[Crawley-Boevey 1991] W. Crawley-Boevey, "Tame algebras and generic modules", Proc. London Math. Soc. (3) 63:2 (1991), 241-265. MR Zbl

[Crawley-Boevey 1992] W. Crawley-Boevey, "Modules of finite length over their endomorphism rings", pp. 127-184 in Representations of algebras and related topics (Kyoto, 1990), edited by H. 
Tachikawa and S. Brenner, London Math. Soc. Lecture Note Ser. 168, Cambridge Univ. Press, 1992. MR Zbl

[Friedlander and Pevtsova 2005] E. M. Friedlander and J. Pevtsova, "Representation-theoretic support spaces for finite group schemes", Amer. J. Math. 127:2 (2005), 379-420. Correction in 128:4 (2006), 1067-1068. MR Zbl

[Friedlander and Pevtsova 2007] E. M. Friedlander and J. Pevtsova, "П-supports for modules for finite group schemes”, Duke Math. J. 139:2 (2007), 317-368. MR Zbl

[Happel 1988] D. Happel, Triangulated categories in the representation theory of finite-dimensional algebras, London Mathematical Society Lecture Note Series 119, Cambridge Univ. Press, 1988. MR Zbl

[Hochster 1969] M. Hochster, "Prime ideal structure in commutative rings", Trans. Amer. Math. Soc. 142 (1969), 43-60. MR Zbl

[Jantzen 2003] J. C. Jantzen, Representations of algebraic groups, 2nd ed., Mathematical Surveys and Monographs 107, American Mathematical Society, Providence, RI, 2003. MR Zbl

[Krause 2003] H. Krause, “A short proof for Auslander's defect formula”, Linear Algebra Appl. 365 (2003), 267-270. MR Zbl

[Neeman 1992] A. Neeman, "The connection between the $K$-theory localization theorem of Thomason, Trobaugh and Yao and the smashing subcategories of Bousfield and Ravenel", Ann. Sci. École Norm. Sup. (4) 25:5 (1992), 547-566. MR Zbl

[Neeman 1996] A. Neeman, “The Grothendieck duality theorem via Bousfield's techniques and Brown representability”, J. Amer. Math. Soc. 9:1 (1996), 205-236. MR Zbl

[Neeman 2001] A. Neeman, Triangulated categories, Annals of Mathematics Studies 148, Princeton Univ. Press, 2001. MR Zbl

[Neeman 2011] A. Neeman, "Colocalizing subcategories of $\mathbf{D}(R)$ ", J. Reine Angew. Math. 653 (2011), 221-243. MR Zbl

[Skowroński and Yamagata 2011] A. Skowroński and K. Yamagata, Frobenius algebras, I: Basic representation theory, European Mathematical Society, Zürich, 2011. MR Zbl

[Suslin 2006] A. Suslin, "Detection theorem for finite group schemes", J. Pure Appl. Algebra 206:12 (2006), 189-221. MR Zbl

[Suslin et al. 1997] A. Suslin, E. M. Friedlander, and C. P. Bendel, "Support varieties for infinitesimal group schemes", J. Amer. Math. Soc. 10:3 (1997), 729-759. MR Zbl

[Waterhouse 1979] W. C. Waterhouse, Introduction to affine group schemes, Graduate Texts in Mathematics 66, Springer, 1979. MR Zbl

Received 14 Apr 2016. Accepted 29 Aug 2016.

DAVE BENSON: d.j.benson@abdn.ac.uk

Department of Mathematics, University of Aberdeen, Meston Building, Aberdeen, AB243UE, United Kingdom

SRIKANTH B. IYENGAR: iyengar@math.utah.edu

Department of Mathematics, University of Utah, 155 South 1400 East, Room 233,

Salt Lake City, UT 84112, United States

HENNING KRAUSE: hkrause@math.uni-bielefeld.de

Fakultät für Mathematik, Universität Bielefeld, PO Box 100131, D-33501 Bielefeld, Germany

Julia Pevtsova: pevtsova@uw. edu

Department of Mathematics, University of Washington, Box 354350, Seattle, WA 98195 ,

United States 


\title{
ANNALS OF K-THEORY
}

\author{
msp.org/akt
}

EDITORIAL BOARD

\author{
Paul Balmer University of California, Los Angeles, USA \\ balmer@math.ucla.edu \\ Spencer Bloch University of Chicago, USA \\ bloch@math.uchicago.edu \\ Alain Connes Collège de France; Institut des Hautes Études Scientifiques; Ohio State University \\ alain@connes.org \\ Guillermo Cortiñas Universidad de Buenos Aires and CONICET, Argentina \\ gcorti@dm.uba.ar \\ Eric Friedlander University of Southern California, USA \\ ericmf@usc.edu \\ Max Karoubi Institut de Mathématiques de Jussieu - Paris Rive Gauche, France \\ max.karoubi@imj-prg.fr \\ Gennadi Kasparov Vanderbilt University, USA \\ gennadi.kasparov@vanderbilt.edu \\ Alexander Merkurjev \\ University of California, Los Angeles, USA \\ merkurev@math.ucla.edu \\ Amnon Neeman amnon.Australian National University \\ neeman@anu.edu.au \\ Jonathan Rosenberg (Managing Editor) \\ University of Maryland, USA \\ jmr@math.umd.edu \\ Marco Schlichting University of Warwick, UK \\ schlichting@warwick.ac.uk \\ Andrei Suslin Northwestern University, USA \\ suslin@math.northwestern.edu \\ Vladimir Voevodsky Institute for Advanced Studies, USA \\ vladimir@math.ias.edu \\ Charles Weibel (Managing Editor) \\ Rutgers University, USA \\ weibel@math.rutgers.edu \\ Guoliang Yu Texas A\&M University, USA \\ guoliangyu@math.tamu.edu \\ PRODUCTION \\ $\begin{array}{ll}\text { Silvio Levy } & \text { (Scientific Editor) } \\ \text { production@msp.org }\end{array}$
}

Annals of K-Theory is a journal of the K-Theory Foundation (ktheoryfoundation.org). The K-Theory Foundation acknowledges the precious support of Foundation Compositio Mathematica, whose help has been instrumental in the launch of the Annals of K-Theory.

See inside back cover or msp.org/ant for submission instructions.

The subscription price for 2017 is US $\$ 420 /$ year for the electronic version, and $\$ 470 /$ year (+\$25, if shipping outside the US) for print and electronic. Subscriptions, requests for back issues and changes of subscriber address should be sent to MSP.

Annals of K-Theory (ISSN 2379-1681 electronic, 2379-1683 printed) at Mathematical Sciences Publishers, 798 Evans Hall \#3840, c/o University of California, Berkeley, CA 94720-3840 is published continuously online. Periodical rate postage paid at Berkeley, CA 94704, and additional mailing offices.

AKT peer review and production are managed by EditFlow ${ }^{\circledR}$ from MSP.

PUBLISHED BY

mathematical sciences publishers nonprofit scientific publishing

http://msp.org/

(C) 2017 Mathematical Sciences Publishers 


\section{ANNALS}

2017

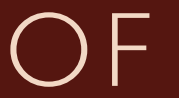

K-THEORY no. 3

Exterior power operations on higher $K$-groups via binary complexes

Tom Harris, Bernhard Köck and Lenny Taelman 